\title{
Event - Related Potentials on the Application of Deception Detection in the Simulation of Concealing Cases
}

\author{
Ruiying Li ${ }^{1,}$, Hongguang Liu ${ }^{1,2}$, Jing Yong ${ }^{1}$ \\ ${ }^{1}$ Institute of Psychological Research, University of International Relations, Beijing, China \\ ${ }^{2}$ Institute of Criminology, Peoples Public Security University of China, Beijing, China
}

Email address:

ruiying_li9586@126.com (Ruiying Li), liuhg93@163.com (Hongguang Liu), yongjing2017@hotmail.com (Jing Yong)

${ }^{*}$ Corresponding author

\section{To cite this article:}

Ruiying Li, Hongguang Liu, Jing Yong. Event - Related Potentials on the Application of Deception Detection in the Simulation of Concealing Cases. American Journal of Applied Psychology. Vol. 6, No. 3, 2017, pp. 42-50. doi: 10.11648/j.ajap.20170603.13

Received: July 14, 2017; Accepted: August 5, 2017; Published: August 14, 2017

\begin{abstract}
This study used Event-related Potentials (ERPs) to examine the difference between three issue types (related, foil, and unrelated issues) and two reaction types (honesty and deception reaction). This was a subject-in design. Two sets of data were collected. First, the behavior data showed that the reaction time of the deception was $200 \mathrm{~ms}$ slower than that of honesty, and the decreasing order of the reaction time on three designed issue types was foil issues, unrelated issues, and related issues. Second, the EEG data showed that related issues produce the most significant ERPs effect; the obvious wave separation of honesty reaction was between $300 \mathrm{~ms}$ to $800 \mathrm{~ms}$ versus $200 \mathrm{~ms}$ to $500 \mathrm{~ms}$ in experiment of deception; the deception reaction could evoke distinct P300, which was most obvious in left parietal lobes such as FC3, C3, CP3, P3; P3 was more sensitive in the time window of $200 \mathrm{~ms}$ to $400 \mathrm{~ms}$, honesty reaction evoked obvious positive wave whereas deception evoked negative wave. In conclusion, the reaction time of deception was significantly slower than honesty. Moreover, deception evoked the obvious P300 effect. Based on the waveform evoked by different issue types, left parietal lobe waveform could be used as judgement of honesty or deception, and the correlation between suspect and the case could be decided.
\end{abstract}

Keywords: ERPs, Simulation of Concealing Cases, Deception Detection, P300

\section{Introduction}

Event - related Potentials (ERPs) technology was used for deception detection, which was extracted from the cognitive potentials related to cognition and memory processing from the ERPs. By recording EEG in the cognitive processing of case relevant content, the relationship between the person taking the test and the case was analyzed. At present, the most common identification index of ERPs deception detection was P300, thus it was also called P300 deception detection technology [10].

ERPs detected the cognitive EEG difference between the related issues and unrelated issues, to examine whether the person know the information is related to the crime or not. It was a direct method of detecting one's psychological activity. The essence of ERPs deception detection technology was based on the analysis of the EEG characteristics of the two cognitive processes: recognition and deception. Lying was accompanied by an increase in cognitive load [4].

In the process of lying, unlike those who tell the truth, liars need to spend more energy to fabricate some stories and details, which requires more cognitive efforts [8]. Lying included a series of cognitive processes such as suppressing honest answers, choosing a false answer, monitoring behaviour, and speculating on others, thus it was considered that lying would lead to an increase in cognitive load [18]. Rosenfeld had made the accuracy rate of $95 \%$ by combining the Concealed Information Test (CIT) with ERPs technology to detect information of concealing crime [22].

Farwell and Donchin found that stimuli associated with spy work led to Memory and Encoding Related Multifaceted Electroencephalographic Response (MERMER) in the experimental group with an incubation period of more than $800 \mathrm{~ms}$, including the positive component P300 with the 
largest amplitude in the parietal lobe and a sub-late negative component with longer latency in the frontal cortex, and the identification of the identity of the subject with MERMER as the distinguishing standard [11]. In some countries, the ERPs technology with MERMER as an indicator has been applied in practice, such as spy recognition, case investigation etc. Farwell uses words and phrases as stimulus to detect the feasibility and accuracy of MERMER as a deception detection tool [10].

\section{Background}

As polygraph allowed the emergence of non-conclusive report, so in some cases, it was not easy to produce a definite conclusion. It was necessary to explore a new distinguishing standard to provide a reference for the results of polygraph. Therefore, this study explored the feasibility and effective identification of ERPs technology in the simulation of concealing cases. If necessary, the combination of the results of the polygraph and the results of ERPs could arrive at a comprehensive conclusion conducive to decisions-making.

\section{Method}

\subsection{Participants}

The paper submitted to the conference should be $6-12$ pages 30 college students participated in the experiment, aged 18-28 years (23.4 \pm 3.6$)$. All subjects were right-handed. They had no brain trauma or nervous system diseases. Their vision or corrected visual acuity was normal. All of them used Chinese as their mother tongue. All subjects were first-time participants in ERPs experiments, and they were told it was harmless physically and mentally before the experiment. Out of 30 participants, 26 produced effective results, including 17 males and 9 females. 4 participants produced invalid results.

\subsection{Tool}

ERPs workstation made by Neuroscan Company was employed in the experiment. The international 40 lead system was used to record EEG.

\subsection{Design}

Experimental design was 3 (problem type: related issues, foil issues, irrelevant issues) $\times 2$ (response: honest, deception) subject-in design. The stimulus followed the sequence of prompt (each group prompts once) - text stimulation - key response. Subjects were asked to make an honest response or deceptive response based the prompts on the screen.

The background material was based on a real concealing case. The experimenter made up a simulation case including a lot of fictitious information, such as time of the crime, the location, communication tools, funds and programs.

The experiment used some short sentences as the stimulating material. The formal experimental stimulus was divided into three categories, namely related issues, foil issues, and irrelevant issues. There were 30 questions, according to the material of the 30 information points compiled, and the content contains information related to person, location, communication tools, funds, and programs. Related issues were linked to the simulation case; foil issues were homogeneous with the relevant issues, but almost impossible to occur; unrelated issues were autobiographical information of the participants or common sense. These issues were expressed in text with less than 10 words. The sequences were GO / NOGO paradigm. That means the total number of stimulus sequences was equal. The subjects were asked to respond according to the prompts on the screen. They were instructed to press two different buttons to make "yes" or "no" as an answer. EEG data was collected from the start of experiment.

In the experiment, each participant was subjected to make both deceptive response and honest response, and the ABBA model was used to balance the experiment. E-prime 2.0 was used to present the stimuli. There were 6-10 Chinese words in each issue. The background was white and the words were black. The distance was about $80 \mathrm{~cm}$. The horizontal viewing angle was $0.84^{\circ}-4.72^{\circ}$, and the vertical viewing angle was $0.52^{\circ}-3.40^{\circ}$. Every issue was presented with the word "yes" or "no" to follow. Every participant accepted 180 stimulus trails. We presented all the questions in a pseudo-random way.

\subsection{Procedure}

A week before the experiment, the participants were asked to sample the contents of the material. They did a case information detection test before the experiment. The formal test procedure was as follows:

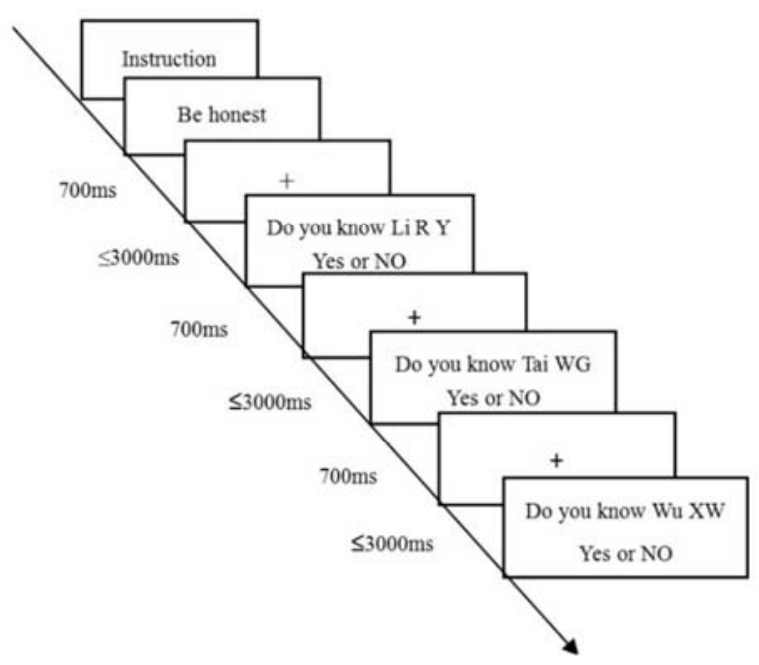

Figure 1. The formal test procedure.

\subsection{Data Collection}

EEG was recorded by the 40-conductive cap of the international 10-20 system. The reference electrode was CZ. Horizontal electro-oculography (HEOG) was recorded on lateral right eye and Vertical electro-oculo graphy (VEOG) was recorded on orbital left eye. Average resistance of the electrode was less than $5 \mathrm{k} \Omega$. Filter bandpass was $0.05 \sim 70 \mathrm{~Hz}$. 
A/D conversion sampling rate was $1000 \mathrm{~Hz} / \mathrm{lead}$. Behavior data was recorded at the same time.

\subsection{Data Analysis}

In the off-line analysis, the reference electrode was converted to bilateral mastoid mean. The experimenter made bandpass filter to be $0.01 \sim 45 \mathrm{~Hz}$ to remove high frequency noise, and used independent component analysis to remove the eye artifacts and other artifacts. The time window of the event analysis was at $0-1000 \mathrm{~ms}$ after stimulation, and the baseline was $200 \mathrm{~ms}$ before stimulation. Data with amplitudes greater than $100 \mathrm{uV}$ were removed automatically as artifacts. According to the cognitive attribute and response type, EEG data was superimposed average and filtered again. According to the waveform and EEG Mapping, 12 electrodes were selected (FC3, FCZ, FC4, C3, CZ, C4, CP3, CPZ, CP4, P3, PZ, P4) to analyze.

\section{Result}

\subsection{Behaviour Data}

Table 1. The reaction time $(\mathrm{ms})(N=26)$.

\begin{tabular}{lll}
\hline Reaction Issue & Honesty $(\boldsymbol{M} \pm \boldsymbol{S D})$ & Deception $(\boldsymbol{M} \pm \boldsymbol{S D})$ \\
\hline Related issues & $1036.36 \pm 157.28$ & $1208.81 \pm 215.54$ \\
Foil issues & $1162.53 \pm 218.50$ & $1308.78 \pm 264.29$ \\
Unrelated issues & $1162.53 \pm 218.50$ & $1304.90 \pm 254.82$ \\
\hline
\end{tabular}

As shown in Table 1 , the experimenter used $3 \times 2$ repeated measurement of variance analysis to analyze the reaction data. The results showed that the main effect of the reaction time was significant, $F(1,25)=49.29, p<0.0001, \eta_{\mathrm{p}}^{2}=0.734$. The reaction time of deception $(1274.16 \pm 244.88 \mathrm{~ms})$ was significantly slower than honesty $(1095.73 \pm 189.47)$. The main effect of the issue type and the interaction of both were not significant, $F(1,25)=2.39, p>0.05 ; F(1,25)=0.65, p>0.05$.

Table 2. The accuracy $(\%)(N=26)$.

\begin{tabular}{lll}
\hline Accuracy Issue & Honesty $(\boldsymbol{M} \pm \boldsymbol{S D})$ & Deception $(\boldsymbol{M} \pm \boldsymbol{S D})$ \\
\hline Related issues & $95.64 \pm 4.60$ & $96.64 \pm 3.74$ \\
Foil issues & $86.00 \pm 10.48$ & $74.35 \pm 11.80$ \\
Unrelated issues & $86.79 \pm 9.59$ & $83.84 \pm 8.72$ \\
\hline
\end{tabular}

As shown in Table 2, the experimenter used $3 \times 2$ repeated measurement of variance analysis to analyze the accuracydata. The results showed that the main effect of the reaction was significant, $F(1,25)=25.65, p<0.001$. The accuracy of deception $(84.62 \% \pm 8.08)$ was significantly lower than honesty $(89.48 \% \pm 8.22)$. The main effect of the issue type was significant, $F(1,25)=28.07, p<0.001, \eta_{\mathrm{p}}^{2}=0.723$. The order was Related issues $(96.14 \% \pm 4.17)>$ Unrelated issues $(85.32 \% \pm 9.16)>$ Foil issues $(85.32 \% \pm 9.16)$. The interaction of both was significant, $F(1,25)=13.25, p<0.001, \eta_{\mathrm{p}}^{2}=0.651$. Simple effect analysis found that two types of reaction differ significantly, $F(2,24)=9.35, p<0.05$. But three types of issues were not different significantly, $F(2,24)=0.23, p=0.713$.

\subsection{EEG Data}

\subsubsection{The EEG Data of Three Issue Types in Honest Reaction}

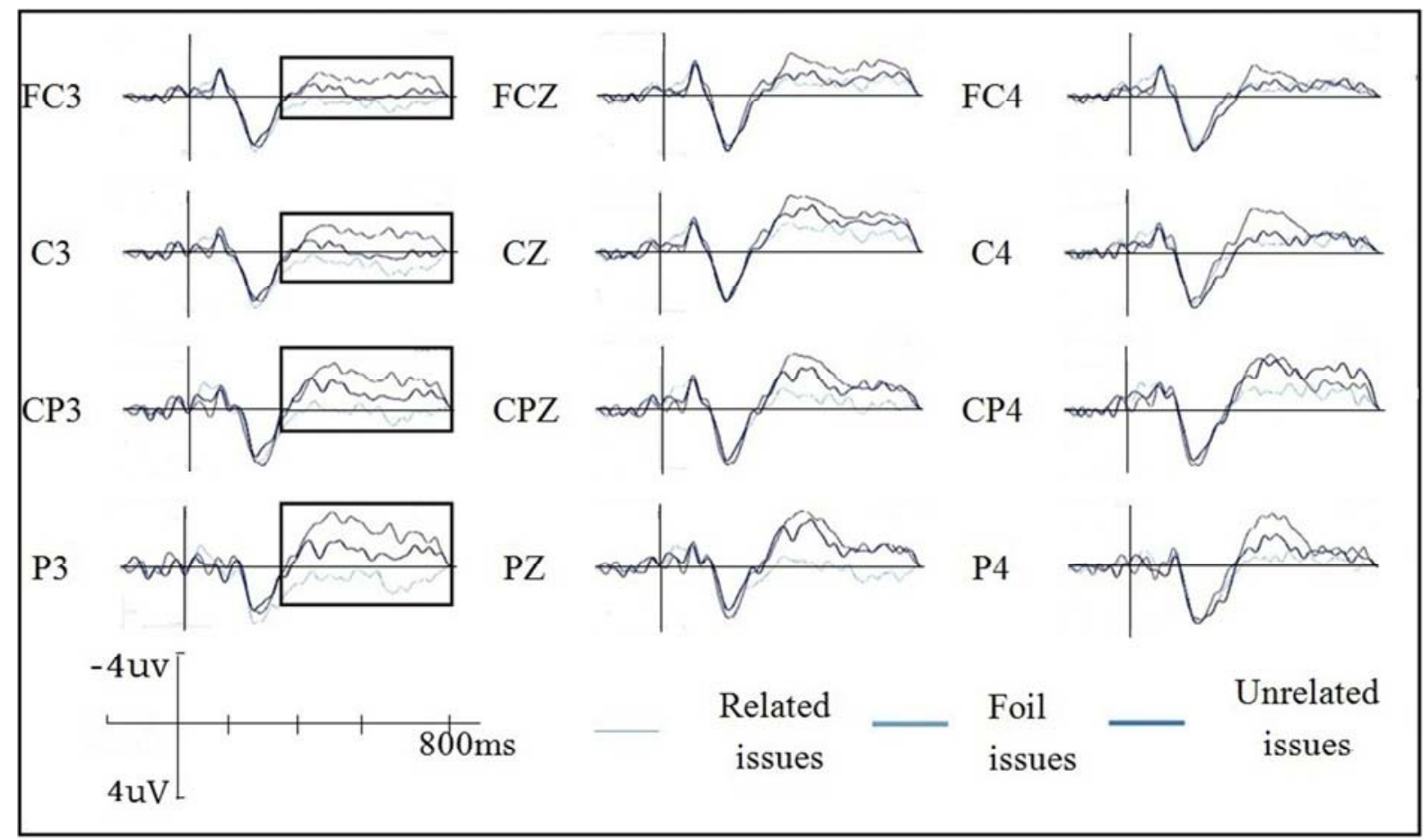

Figure 2. The ERPs waveform of 3 issue types in honest response. 
As shown in Figure 2, honest response induced a positive component on $100-300 \mathrm{~ms}$, and the wave separation appeared on $300-800 \mathrm{~ms}$. The experimenter used 3 issue types (related issues, foil issues, and unrelated issues) $\times 3$ (hemisphere left [FC3, C3, CP3, P3], centre [FCZ, CZ, $\mathrm{CPZ}, \mathrm{PZ}]$, right [FC4, $\mathrm{C} 4, \mathrm{CP} 4, \mathrm{P} 4])$, amplitude variance showed measurements to analyze the average waveform on 300-800ms.

The results showed that the main effect of the issue type was significant, $F(2,24)=5.616, p<0.05, \eta_{\mathrm{p}}^{2}=0.652$. Foil issues $(-1.728 \pm 0.132 \mu \mathrm{V})$ were more negative than the related issues $(-0.457 \pm 0.366 \mu \mathrm{V})$ and unrelated issues
$(-1.728 \pm 0.132 \mu \mathrm{V})$. The main effect of hemispheric effect was significant, $F(2,24)=42.603, p<0.0001, \eta_{\mathrm{p}}^{2}=0.934$. The order was left hemisphere $(-0.015 \pm 0.330 \mu \mathrm{V})>$ right hemisphere $(-1.365 \pm 0.155 \mu \mathrm{V})>$ midline $(-2.601 \pm 0.159$ $\mu \mathrm{V})$. The interaction between the type of issue and the hemisphere was significant, $F(4,48)=10.642, p<0.001, \eta_{\mathrm{p}}^{2}$ $=0.780$. Simple effect analysis found that three types of issues were significant different in the left hemisphere, $F(2$, $24)=17.35, p<0.005$. But it was not significant in the centre or right hemisphere, $F(2,24)=0.13, p=0.883 ; F(2,24)$ $=1.50, p=0.296$.

\subsubsection{The EEG Data of Issue Types in Deception Reaction}

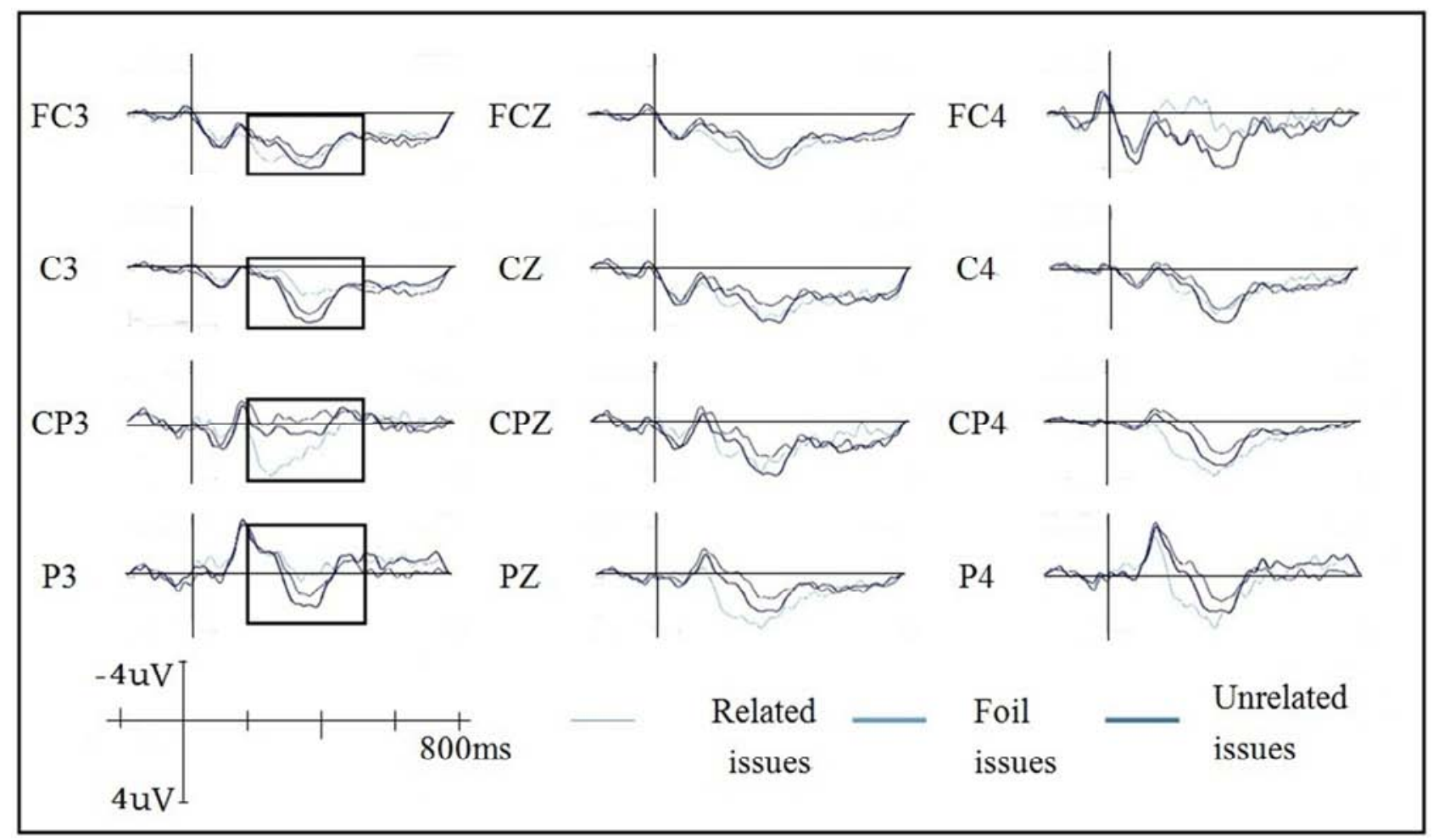

Figure 3. The ERPs waveform of 3 issue types in deceptive response.

As shown in Figure 3, Deceive reaction induced a positive component on $200-500 \mathrm{~ms}$. The experimenter used 3 issue types (related issues, foil issues and unrelated issues) $\times 3$ hemisphere (left [FC3, C3, CP3, P3], centre [FCZ, CZ, CPZ, $\mathrm{PZ}$, right [FC4, C4, CP4, P4]), to analyze summit waveform in the time window of $200-500 \mathrm{~ms}$ by amplitude variance repeated measurements.

The results showed that the main effect of the issue type was not significant, $F(2,24)=1.358, p=0.326, \eta_{\mathrm{p}}^{2}=0.312$. The main effect of hemispheric effect was significant, $F(2,24)$ $=5.778, p<0.05, \eta_{\mathrm{p}}^{2}=0.658$. The order was left hemisphere $(5.455 \pm 0.834 \mu \mathrm{V})>$ right hemisphere $(4.881 \pm 0.379 \mu \mathrm{V})>$ midline $(3.948 \pm 0.787 \mu \mathrm{V})$. The interaction between the type of issue and the hemisphere was not significant, $F(4,48)$ $=2.166, p=0.135, \eta_{\mathrm{p}}^{2}=0.419$. 


\subsubsection{The EEG Data of Related Issue in Honesty and Deceptive Reaction}

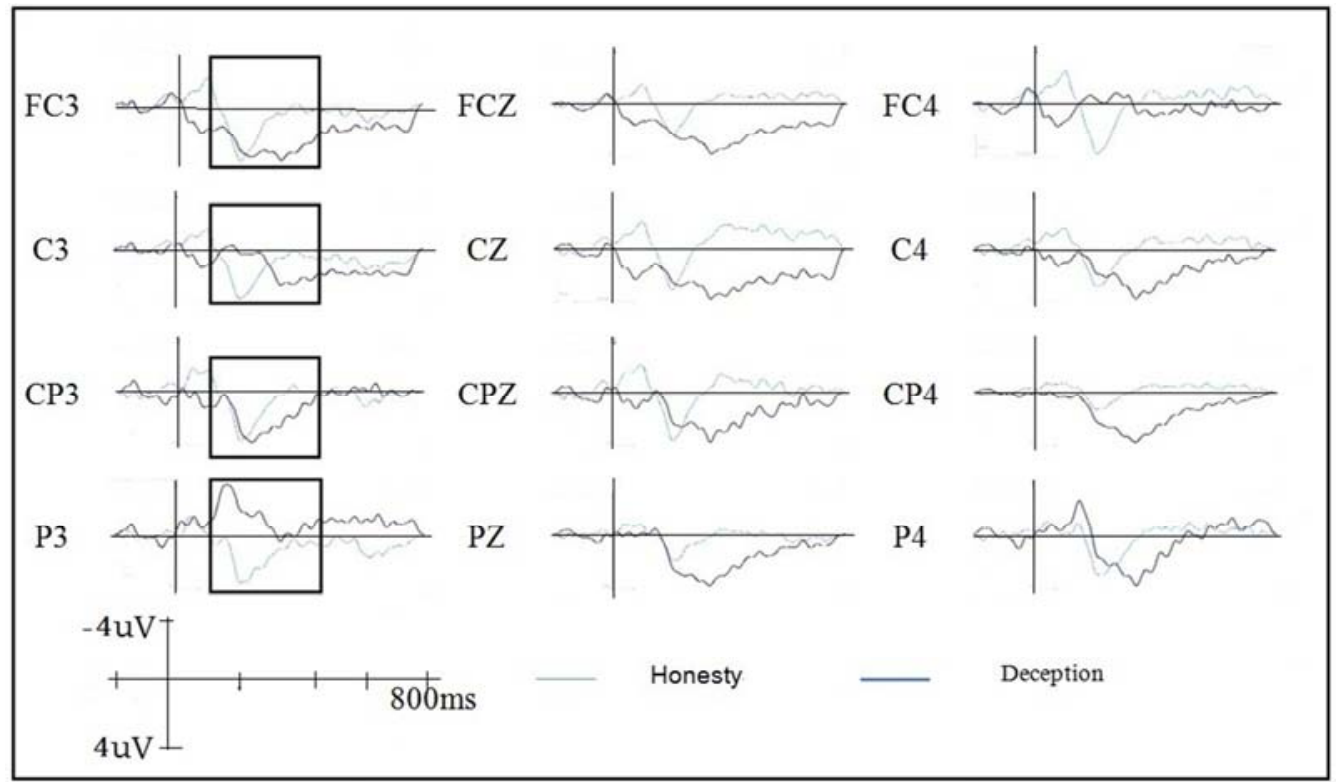

Figure 4. The ERPs waveform of related issue in honesty and deceptive response.

As shown in Figure 4, the deceptive response produces a significant P300 effect with latency greater than $300 \mathrm{~ms}$.

In the time window of $100-500 \mathrm{~ms}$, the experimenter used 2 (reaction types: honesty, deception) $\times 3$ (hemisphere: left $[\mathrm{FC} 3$, $\mathrm{C} 3, \mathrm{CP} 3, \mathrm{P} 3]$, centre [FCZ, CZ, CPZ, PZ], right [FC4, C4, $\mathrm{CP} 4, \mathrm{P} 4]$ ), to analyze summit of waveform of related issue by amplitude variance repeated measurements. The results showed that the main effect of the reaction type, hemisphere and their interaction were not significant, $F(1,25)=0.275, p=$ $0.636, \eta_{\mathrm{p}}^{2}=0.084 ; F(2,24)=1.324, p=0.334, \eta_{\mathrm{p}}^{2}=0.306$; $F(2,24)=2.018, p=0.214, \eta_{\mathrm{p}}^{2}=0.402$.
The experimenter used amplitude variance repeated measurements to analyze the latency of related issue by 2 (reaction types: honesty, deception) $\times 3$ (hemisphere : left [FC3, C3, CP3, P3], centre [FCZ, CZ, CPZ, PZ], right [FC4, $\mathrm{C} 4, \mathrm{CP} 4, \mathrm{P} 4])$. The results showed that the main effect of the reaction type was significant, $F(1,25)=27.928, p<0.05, \eta_{\mathrm{p}}^{2}$ $=0.903$. The latency of deceptive response $(301.50 \pm$ $18.01 \mathrm{~ms})$ was longer than honesty $(208.42 \pm 0.48 \mathrm{~ms})$. The main effect of hemisphere and their interaction of reaction type and hemisphere were not significant, $F(2,24)=0.529, p$ $=0.614, \eta_{\mathrm{p}}^{2}=0.150 ; F(2,24)=0.582, p=0.588, \eta_{\mathrm{p}}^{2}=0.162$.

\subsubsection{The EEG Data of Foil Issue in Honesty and Deceptive Reaction}

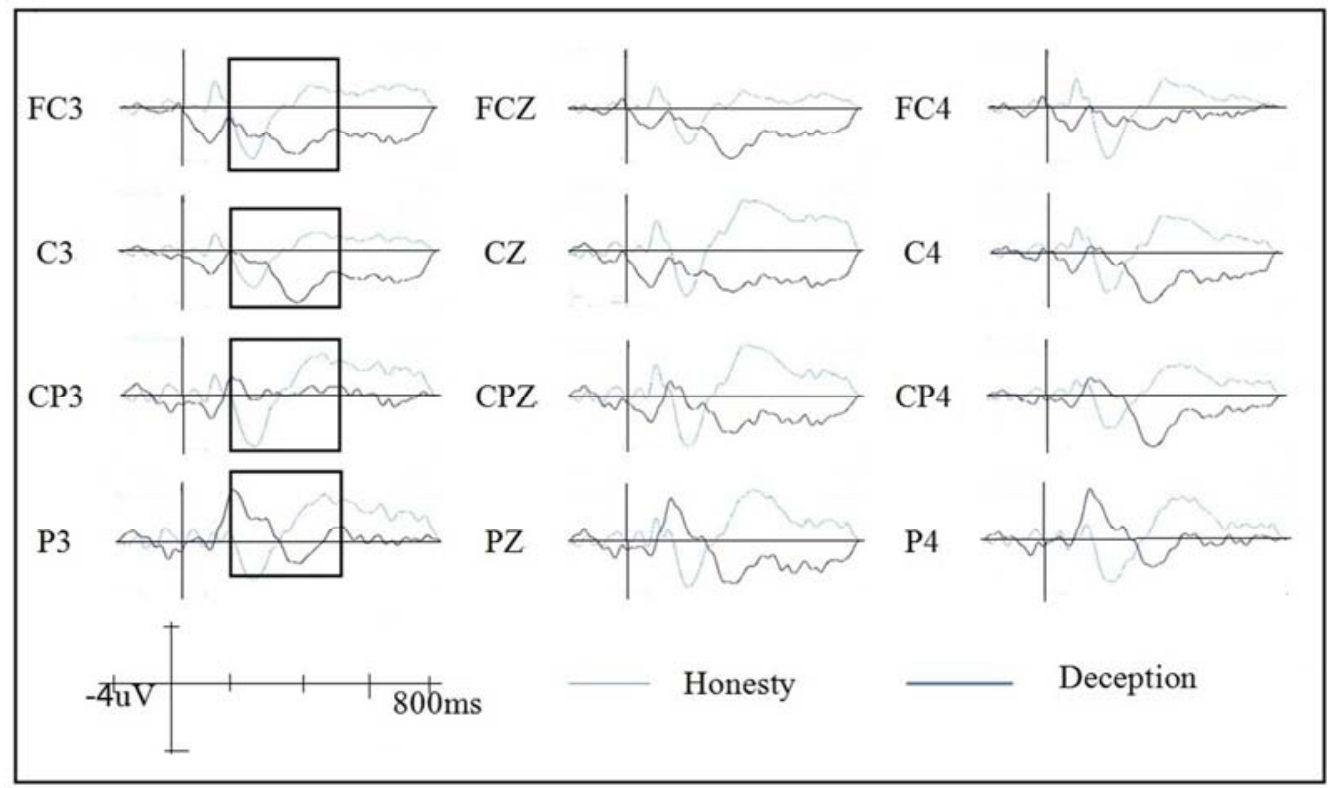

Figure 5. The ERPs waveform of foil issue in honesty and deceptive response. 
As shown in Figure 5, the deceptive response produces a significant P300 effect with latency greater than $300 \mathrm{~ms}$.

In the time window of $100-500 \mathrm{~ms}$, the experimenter used 2 (reaction types: honesty, deception) $\times 3$ (hemisphere: left [FC3, $\mathrm{C} 3, \mathrm{CP} 3, \mathrm{P} 3]$, centre [FCZ, CZ, CPZ, PZ], right [FC4, C4, $\mathrm{CP} 4, \mathrm{P} 4]$ ), to analyze summit of waveform of foil issue by amplitude variance repeated measurements. The results showed that the main effect of the reaction type, hemisphere and their interaction were all not significant, $F(1,25)=0.856, p$ $=0.423, \eta_{\mathrm{p}}^{2}=0.222 ; F(2,24)=0.064, p=0.939, \eta_{\mathrm{p}}^{2}=0.021 ; F$ $(2,24)=0.068, p=0.935, \eta_{\mathrm{p}}^{2}=0.022$.
The experimenter used amplitude variance repeated measurements to analyze latency of foil issue, by 2 (reaction types: honesty, deception) $\times 3$ (hemisphere: left [FC3, C3, CP3, $\mathrm{P} 3]$, centre [FCZ, CZ, CPZ, PZ], right [FC4, C4, CP4, P4]). The results showed that the main effect of the reaction type was significant, $F(1,25)=15.714, p<0.05, \eta_{\mathrm{p}}^{2}=0.840$, and the latency of deceptive response $(315.83 \pm 25.73 \mathrm{~ms})$ is longer than honesty $(215.00 \pm 2.49 \mathrm{~ms})$. The main effect of hemisphere and the interaction of reaction type and hemisphere were not significant, $F(2,24)=0.304, p=0.749$, $\eta_{\mathrm{p}}^{2}=0.092 ; F(2,24)=0.761, p=0.507, \eta_{\mathrm{p}}^{2}=0.202$.

\subsubsection{The EEG Data of Unrelated Issue in Honesty and Deceptive Reaction}

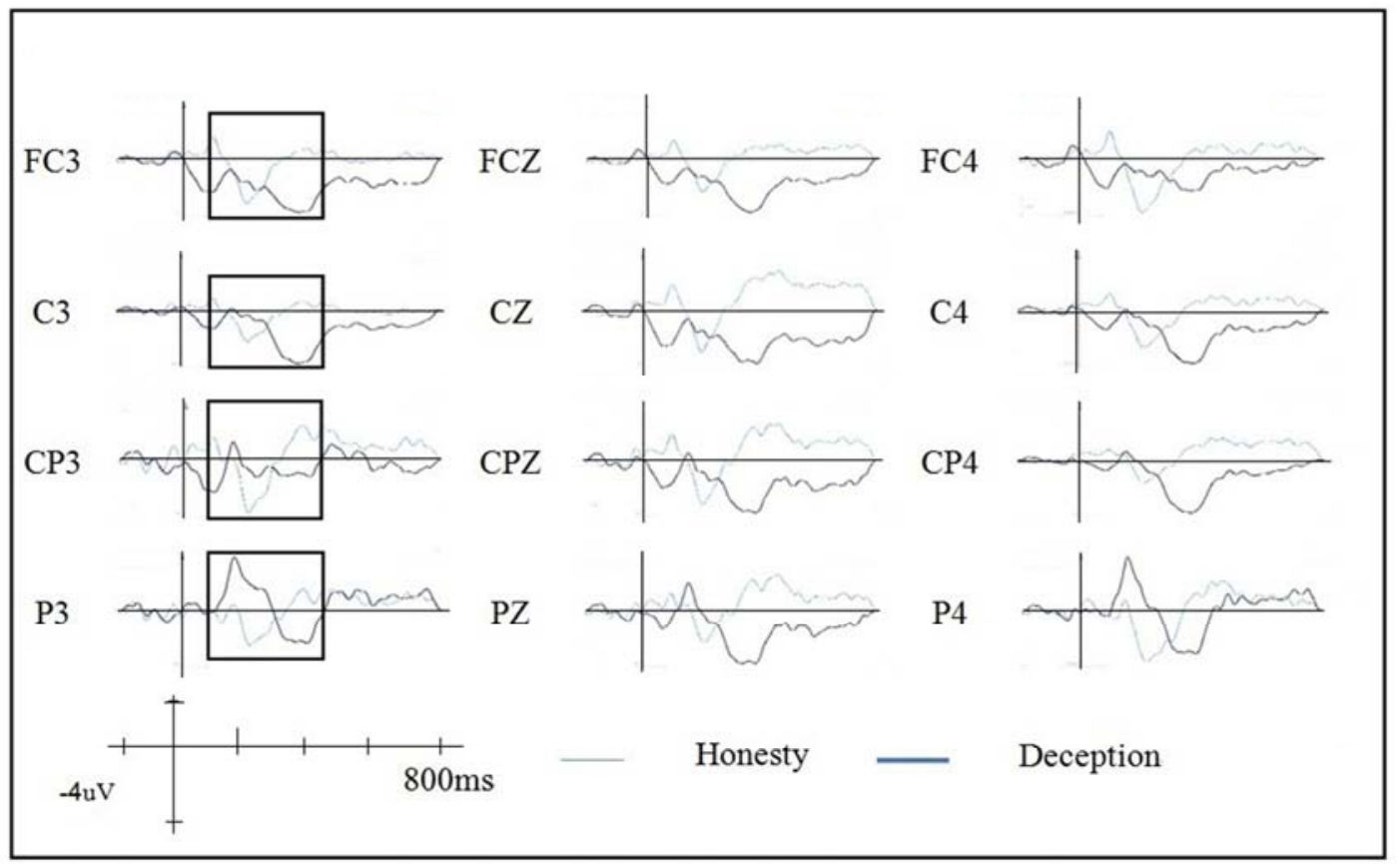

Figure 6. The ERPs waveform of unrelated issue in honesty and deceptive response.

As shown in the Figure 6, the deceptive response produces a significant P300 effect with latency greater than $300 \mathrm{~ms}$.

In the time window of $100-500 \mathrm{~ms}$, the experimenter used 2 (reaction types: honesty, deception) $\times 3$ (hemisphere: left [FC3, $\mathrm{C} 3, \mathrm{CP} 3, \mathrm{P} 3]$, centre [FCZ, CZ, CPZ, PZ], right [FC4, C4, $\mathrm{CP} 4, \mathrm{P} 4]$ ), to analyze summit of waveform of unrelated issue by amplitude variance repeated measurements. The results showed that the main effect of the reaction type, hemisphere and their interaction were all not significant, $F(1,25)=0.03, p$ $=0.874, \eta_{\mathrm{p}}^{2}=0.010 ; F(2,24)=1.156, p=0.376, \eta_{\mathrm{p}}^{2}=0.278$; $F(2,24)=0.785, p=0.498, \eta_{\mathrm{p}}^{2}=0.207$.

The experimenter used amplitude variance repeated measurements to analyze latency of unrelated issue, by 2 (reaction types: honesty, deception) $\times 3$ (hemisphere: left [FC3, $\mathrm{C} 3, \mathrm{CP} 3, \mathrm{P} 3]$, centre [FCZ, CZ, CPZ, PZ], right [FC4, C4, $\mathrm{CP} 4, \mathrm{P} 4])$. The results showed that the main effect of the reaction type was significant, $F(1,25)=40.563, p<0.01, \eta_{\mathrm{p}}^{2}=$ 0.931 , and the latency of deceptive response $(346.92 \pm 22.21 \mathrm{~ms})$ was longer than honesty $(205.92 \pm 1.78 \mathrm{~ms})$. The main effect of hemisphere and the interaction of reaction type and hemisphere were not significant, $F(2,24)=1.118, p=0.387, \eta_{\mathrm{p}}^{2}$ $=0.271 ; F(2,24)=0.825, p=0.483, \eta_{\mathrm{p}}^{2}=0.216$.

\section{Discussion}

\subsection{Behavior Response of Deceptive Reaction and Unfamiliar Stimulus Made Delay Effect}

The reaction time of foil issue was slower than related issue and unrelated issue, and the accuracy of foil issue was lower than related issue and unrelated issue. The response of the deception was significantly slower than the honest, and the accuracy of deception was significantly lower than the honest.

When the autobiographical information of the participants or common sense (unrelated issue) and case-related information (related issue) were presented, the related memory was easier to be activated. These messages were easier for individual to compare, judge, and reflect. In contrast, 
when the case of foil information (foil issue) was presented, it was the information that individual' memory system had never coded, so more reaction time was needed. In the case of deception, individual added additional psychological processes to suppress the tendency of honest reaction. The difference in behavioral response reflected the cumulative output of the two different execution processes.

Individuals had two conflicting reactions in their mind before the deception reaction. The first was the tendency to response honestly, and the second was the tendency to deceive. The implementation of the deception reaction required an additional process, that was, to suppress the tendency of honest reaction, and to make the implementation of deception. The most important process of deception reaction was to coordinate and control the conflict between the honest response and deception response tendencies. Individuals' brain should compare the two trade-offs, and then make choices. At last, individuals performed a fraud response to make their own behavior in line with the initial plan and objectives.

In other words, deceptive response has two specific psychological processes: the first was to conceal the information stored in the brain, and the second was to perform a deceptive behavior. The completion of the deception response included a more complex execution process. These processes may be the main reason of differences in behavior data between deceptive and honest responses.

In addition, the research of conflict control found that, compared with the task of no reaction to the conflict, when the subjects responded with the task of conflict, the reaction time became slower, and the accuracy was lower. It was the same as the implementation of the deception reaction [9].

\subsection{The P300 Effect of Deceptive Response}

The volatility of P300 may indicate the amount of mental load or brain resources put into the task [24]. The deafness response induced a significant $\mathrm{P} 300$ with latency greater than $300 \mathrm{~ms}$. In the time window of $100-500 \mathrm{~ms}$, there was a significant difference between honest response and deceptive response. As long as individual deceived, there would be additional control process. In fact, the deception reaction was the inhibition of honest reaction. Individuals should not only complete the main task of honest response, but also fight with the advantages of honesty response tendencies effectively. In this process, implementation of individuals' brain played an important role. The complete process was: Identifying the information, inhibiting the honesty response, fighting with the conflict of honesty and deception, creating the intention of deception, and performing deceptive reactions. These series of processes required an effective implementation of the control process to guide and supervise, to control and coordinate the instinctive behavior, and to ensure the successful implementation of fraud.

\subsection{The Category Effect of Different Stimulus Attribute Issue}

As shown in the experimental results, in the time window of $300-800 \mathrm{~ms}$, the three types of issues in the honest response were significantly different in the left hemisphere. According to the "Context updating model" proposed by Donchin, the environmental information was stored in the human brain in a certain way, which was called characterization, namely the database that individuals need to engage in cognitive activities [7]. When a stimulus was presented, the brain recognized and encoded it. Then according to the significance of the task, the brain integrated it into the existing representation to form a new representation. Next, individuals corrected his existing background to adjust the strategy to cope with the future. Amplitude can be regarded as indicators of contextual update in working memory. Compared with the case-related information, the degree of scenario update was more difficult when dealing with the new stimulus such as the foil information, thus the corresponding amplitude was higher.

In the case of deception, the amplitude of the issue type was not significantly different. According to the Cognition Load Theory, an individual's cognitive resources were limited [26]. The more complex the task, the more cognitive resources they would consume. So when the subjects plan to deceive, they would confirm the necessary content based on clear judgement and make the opposite choice. This was complex processing that caused a significant increase in brain cognitive load. That was the reason why the separation of amplitude induced by three kinds of issues occurred. The different stimulus attributes different psychological significance to the subjects, even if they attempted to control all kinds of physical and mental activities consciously, for concealing, but they could not inhibit the different cognitive content in the brain caused by the different stimulus. When the stimuli that contain case-related information were presented, they activated the established information about individuals' brain storage automatically. That was the processing that participants try their best to control consciously. So it induced a change in brain potential that was different from those without this information.

\subsection{Significant Difference Between Honesty and Deception in the Left Posterior Parietal Lobe}

On the left posterior parietal lobe, especially in the electrode point P3, there was significant difference between honest and deceptive reaction. 


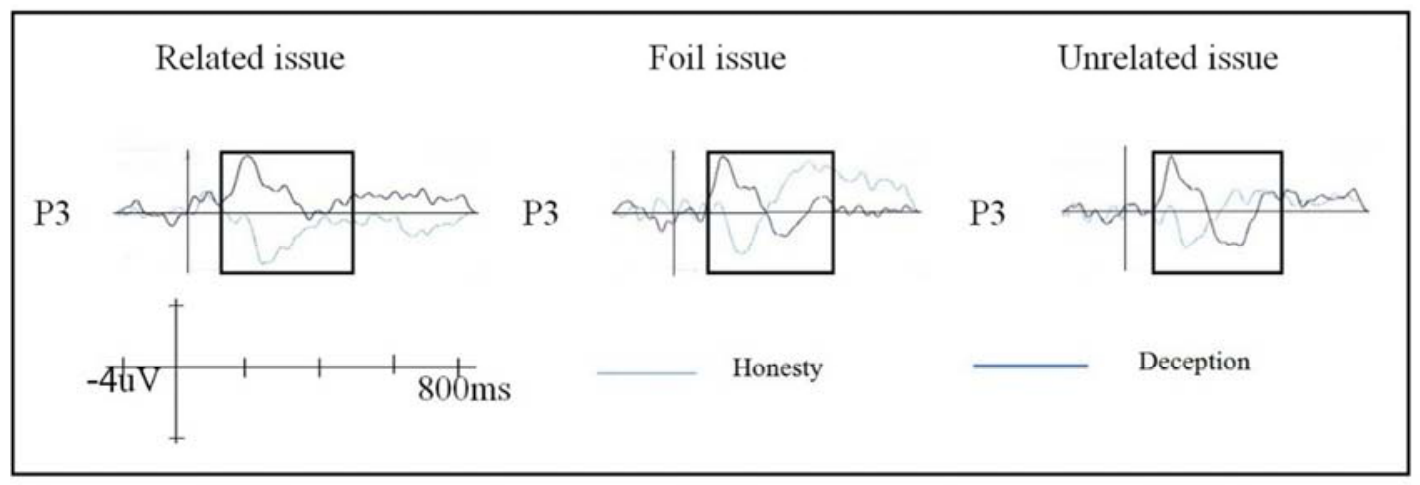

Figure 7. The ERPS waveform of $P 3$ in honesty and deceptive reaction.

As shown in Figure 7, no matter which kind of issues, when it was honest, there was obvious positive wave on $200 \mathrm{~ms}$ and then the waveform return to baseline. But when it was deceptive, it was an obvious negative wave on $180 \mathrm{~ms}$ and a significant positive wave on $360 \mathrm{~ms}$ following. That was an observable phenomenon that the honest response induced P200, while the deceptive reaction induced the wave like N200 and significant P300. The volatility of P300 was positively related to the amount of mental resources invested [3]. P300 reflected the time required to evaluate the difference of stimulus, and its amplitude reflects the renewal of the working memory characterization. P300 was related to the hippocampal structure of the parietal lobe deep margin system [12]. The parietal lobe was associated with the storage of working memory [23]. Research from PET found that the posterior lobe was activated at the storage conditions, and that the left parietal cortex was activated in most of the word working memory tasks [16].

\subsection{The Possibility of ERPs to Be the Effective Screening Tool for the Concealing Cases}

The increase in cognitive load in concealing cases made the characteristic brain potentials that were associated with information related to the case. Compared with other formal cases such as larceny case, the concealing case was often more secret action. In order to leave without traces, such cases were often prepared after a long period of careful planning. The perpetrators used the means as difficult as possible to avoid the police and even without obvious evidence. In this process, the criminal would invest more psychological resources inevitably. In this way, the relevant information must be in deep-coding. In the ERPs test, the case-relevant information would carry out more cognitive processing. For the "criminals", their cognitive load was conducive to the introduction of the relevant information which was related to the characteristics of the brain potential.

When the tests showed both of case-related information and case-unrelated information, the two kinds of stimulus information involved in the processing of different depth, and induced different self-interest. Even if the individual attempted to cheat and disguise, they cannot inhibit the brain cognitive processing of different content brought about by the different brain response directly. When an individual was concerned in the test, on one hand, his fear of the crime was exposed and sanctioned by the law, on the other hand, he was afraid of his identity exposure. So the more sensible the criminals, the more anxious they were. In order to evade his responsibility, he must improve the alertness to the crime, and concealed the real information that he had known to deny the fact. When the criminal choose the reaction which was beneficial to himself, so as to deceive.

When individuals were faced with stimulus that was related to their case, their cognitive preparation enhanced the degree of psychological warning. The level of information was related to the level of monitoring. So the criminal's motivation of passing the test would be higher. Because the state of response with a deceptive intention provoked more cognitive control, it generated more additional motivations. Compared with the honest response, many of the prefrontal cortex associated with cognitive processing increased the activity of the brain, so that the activity level of brain changes, which induced different brain potential. In this way, ERPs produced the possibility to apply for the deception detection in the concealing cases.

\section{Conclusion}

Firstly, the response of the deception reaction was significantly slower than the honest response;

Secondly, simulation of concealing cases induced a significant P300 in the deceived state;

Thirdly, honest or deceptive response was determined by wave characteristics of left posterior lobe. In the time window of 100-400mson P3, honest response induced significant wave, but deceptive reaction induced a significant negative wave first and then there was a significant positive wave;

Lastly, according to waveform separation induced by different types of issues, we could judge the relationship between the person and the case. If the individual made honest response, the more familiar to the information being presented, the smaller amplitude produced; oppositely, the more stranger to the information, the greater amplitude produced. But if the person was deceptive, the wave separation would not be obvious. 


\section{References}

[1] Ambach, W., Bursch, S., Stark, R., \& Vaitl, D. (2010). A concealed information test with multimodal measurement. International Journal of Psychophysiology, 75 (3), 258-267.

[2] Alsufyani, A., Zoumpoulaki, A., Filetti, M., \& Bowman, H. (2013). A new method for detecting deception in event related potentials using individual-specific weight templates. $B M C$ Neuroscience, 14 (1), 1-2.

[3] Brown, C. R., Clarke, A. R., \& Barry, R. J. (2007). Auditory processing in an inter-modal oddball task: effects of a combined auditory/visual standard on auditory target erps. International Journal of Psychophysiology, 65 (2), 122-131.

[4] Cao, B, L., Liu, H, J., Lin, C, D.(2005). Cognitive Load Influenced the Pupill's Strategies Selection of Distributing Working Memory Resources. Psychological Development and Education, 21 (1), 36-42.

[5] Chen, Y, L., Sun, L, B. (2015). Advanced tutorial of Polygraph, Beijing: Press of People's Public Security University of China.

[6] Cui, Q., Zhang, Q, L., Qiu, J., Liu, Q., Du, X, M., Ruan, X, L. (2009). The Functionally Separation of P300 and CNV in Lie Detection. Acta Psychologica Sinica, 41 (4), 316-328.

[7] Donchin, E., (1985). Surprise!--Surprise? Psychophysiology, $22(5), 497$.

[8] DePaulo, B. M., Lindsay, J. J., Malone, B. E., Muhlenbruck, L., Charlton, K., \& Cooper, H. (2003). Cues to deception. Psychological Bulletin, 129 (1), 74.

[9] Fang, F., Liu, Y., \& Shen, Z. (2003). Lie detection with contingent negative variation. International Journal of Psychophysiology, 50 (3), 247-255.

[10] Farwell, L. A. (2012). Brain fingerprinting: a comprehensive tutorial review of detection of concealed information with event-related brain potentials. Cognitive Neurodynamics, 6 (2), 115.

[11] Farwell, L. A., \& Donchin, E. (1991). The truth will out: interrogative polygraph ("lie detection") with event-related brain potentials. Psychophysiology, 28 (5), 531-547.

[12] Friederici, A. D., Mecklinger, A., Spencer, K. M., Steinhauer, K., \& Donchin, E. (2001). Syntactic parsing preferences and their on-line revisions: a spatio-temporal analysis of event-related brain potentials. Cognitive Brain Research, 11 (2), 305-323.

[13] Jang, K. W., Kim, D. Y., Cho, S., \& Lee, J. H. (2013). Effects of the combination of p3-based GKT and reality monitoring on deceptive classification. Frontiers in Human Neuroscience, 7 (2), 18.

[14] Jennifer M. C. Vendemia PhD, Robert F. Buzan MA, Eric P. Green MA, \& Michael J. Schillaci PhD. (2005). Effects of preparedness to deceive on ERP waveforms in a two-stimulus paradigm. Journal of Neurotherapy, 9 (3), 45-70.

[15] Jongsma, M. L., Desain, P., \& Honing, H. (2004). Rhythmic context influences the auditory evoked potentials of musicians and non-musicians. Biological Psychology, 66 (2), 129-152.
[16] Jonides, J., Smith, E. E., Marshuetz, C., Koeppe, R. A., \& Reuterlorenz, P. A. (1998). Inhibition in verbal working memory revealed by brain activation. Proceedings of the National Academy of Sciences of the United States of America, 95 (14), 8410-3.

[17] Jr, J. R., Barnhardt, J., \& Zhu, J. (2005). Differential effects of practice on the executive processes used for truthful and deceptive responses: an event-related brain potential study. Cognitive Brain Research, 24 (3), 386-404.

[18] Langleben, D. D., Schroeder, L., Maldjian, J. A., Gur, R. C., Mcdonald, S., \& Ragland, J. D., et al. (2002). Brain activity during simulated deception: an event-related functional magnetic resonance study. Neuroimage, 15 (3), 727-732.

[19] Liao, S, Z. (1996). Event-related potential and Lie Detection. Journal of Clinical Electroencephalology, 5 (3), 186-188.

[20] Liu, H, G. (2010). Brain Cognitive Progressive Process Analysis of Case Related Information. Journal of People's Public Security University of China (Natural Science Edition), 3, 47-52.

[21] Meegan, D. V. (2008). Neuroimaging techniques for memory detection: scientific, ethical, and legal issues. Am J Bioeth, 8 (1), 9-20.

[22] Rosenfeld, J. P., Labkovsky, E., Winograd, M., Ming, A. L., Vandenboom, C., \& Chedid, E. (2008). The complex trial protocol (ctp): a new, countermeasure-resistant, accurate, p300-based method for detection of concealed information. Psychophysiology, 45 (6), 906-919.

[23] Sun, L, N., Zhou, R, H., \& Li, S, Y. (2010). Study on the Difference of Continuous Working Memory Ability By P300. Chinese Journal of Applied Physiology, 26 (1), 76-76.

[24] Sachs, G., Anderer, P., Margreiter, N., Semlitsch, H., Saletu, B., \& Katschnig, H. (2004). P300 event-related potentials and cognitive function in social phobia. Psychiatry Research Neuroimaging, 131 (3), 249-261.

[25] Sun, D., Chan, C. C., \& Lee, T. M. (2012). Identification and classification of facial familiarity in directed lying: an ERP study. Plos One, 7 (2), e31250.

[26] Sweller, J. (1988). Cognitive load during problem solving: effects on learning. Cognitive Science, 12 (2), 257-285.

[27] Xiong, Y., Luo, Y., Huang, W., Zhang, W., Yang, Y., \& Gao, J. (2014). A novel classification method based on ica and elm: a case study in lie detection. Bio-medical materials and engineering, 23 (23), S357-S363.

[28] Saldžiūnas, V., \& Kovalenka, A. (2012). Polygraph screening in Lithuania and Russia. Polygraph. 41 (4).

[29] Xu, J., Liu, H, G. (2011). Cognitive EEG Lie Detection of Bribery Crime Investigation by "one to one". Journal of Gansu Vocational College of Political Science and Law, 3, 24-27.

[30] Zhao, D., Yang, Q, W., Luo, Y, J. (2015). The Application of Event - related Potential in Lie Detection. Journal of Chengdu Medical College, 10 (4), 500-503.

[31] Zhu, Q., Luo, Y., Chen, H. Liang, H. (2014). Research Orientation of Conceal Information Testing Based on Event related Single Potential. Journal of Xi Nan University (Social Science Edition), 1 (1), 68-73. 